\title{
Failed anti-reflux surgery: Causes and management
}

\author{
Hany Rafik', MD; Amr Kamel' 1 , MD; Ahmed Nafei ${ }^{1}, M D ;$ M. Magdy', MD; \\ Ibrahim N. Elwardany², MD
}

\author{
1) Department of General Surgery, Ain Shams University, Cairo, Egypt. \\ 2) Department of General Surgery, Misr University for Science and Technology, \\ Cairo, Egypt.
}

Background: Failure of anti-reflux surgery means persistence of the previous symptoms of gastro-oesophageal reflux disease (GERD) or appearance of new symptoms of over correction after surgery. This needs a careful evaluation \& management. The aim of this study is the diagnosis of the causes of failed anti-reflux surgery and to select patients who are in need for re-do surgery.

Patients and methods: Over a 31-month period (April 2009 to October 2011), 31 patients (mean age $39.44 \pm 7.5$ ) presented with symptoms of failed anti-reflux surgery starting shortly after the primary surgery. 18 patients (58.1\%) were complaining of symptoms of recurrence and 13 patients $(41.9 \%)$ were complaining of symptoms of overcorrection. Conservative management was tried first and the integrity of the previous fundoplication was evaluated by upper endoscopy, barium oesophago-gastrogram, manometric studies and 24-hours ambulatory pH monitoring.

Results: 11 patients (35.4\%) were managed conservatively, 4 patients (12.9\%) required oesophageal dilatation and 16 patients (51.6\%) were in need for revision of the primary surgery. Improvement was noted in all patients with redo surgery, only 3 patients developed postoperative dysphagia that was managed conservatively.

Conclusion: The rate of redo surgery may be further reduced by a meticulous surgical technique; redo surgery should be offered for only selected patients with failed anti-reflux surgery and should be done by an experienced surgeon to ensure the best results.

Key words: Gastroesophageal reflux disease, fundoplication, re-do.

\section{Introduction:}

Laparoscopic fundoplication became the gold standard in the surgical therapy of GERD. In comparison with open procedures, laparoscopic antireflux surgery has a lower morbidity rate, a better early and late postoperative outcome and is more costeffective. Laparoscopic 360 degrees Nissenfundoplication with crurorrhaphy is the standard procedure, whereas the 270 degrees Toupet technique is the technique of choice for oesophageal motility disorders. ${ }^{1}$

Despite the variety of surgical operations and strategies employed, the overall failure rate is constant at approximately $11 \%-14 \%$ and almost $4 \%-7 \%$ of dissatisfied patients require a re-operation. ${ }^{2}$
Failure of anti-reflux surgery means persistence of the previous symptoms or appearance of new symptoms after surgery and this needs a careful evaluation. Investigations should be directed at both the original preoperative evaluation and current situation. Esophageal manometry might show borderline function predisposing to persistent symptoms postoperatively. The operative report should be obtained and reviewed, with attention to such details as esophageal length, use of a dilator to size a wrap, closure of the hiatus, and division of the short gastric vessels. ${ }^{3}$

Unsuccessful results of surgery for GERD whether associated with hiatal hernia ( $\mathrm{HH})$ or not, is a frustrating event for the surgeon, 
but far more so for the patient because of the well-known poor quality of life. ${ }^{4}$

The aim of this study is the diagnosis of the causes of failed anti-reflux surgery and to select patients who are in need for re-do surgery.

\section{Patients and methods:}

This prospective, randomized study was conducted in Ain Shams university hospitals from April 2009 to October 2011. A total of 31 adult patients with failed anti-reflux surgery shortly after the primary surgery within the first 1-3 months were included in the study. Eleven patients (35.5\%) were with previous laparoscopic fundoplication and twenty patients (64.5\%) were with previous open fundiolication.

These patients were assessed for either recurrence of symptoms of reflux or appearance of new symptoms which were symptoms of overcorrection such as dysphagia and gas-bloat syndrome, and then the need for repeated surgery was assessed. Any patient with preoperative dysphagia was excluded from the study.

These patients were classified into two groups. The $1^{\text {st }}$ group who complained of recurrence of symptoms and the $2^{\text {nd }}$ group who complained of symptoms of overcorrection like dysphagia and gas bloating syndrome.

The diagnosis of recurrence was made in out patient clinic on basis of typical history including recurrent heart burn and regurgitation or appearance of new symptoms like laryngitis, asthma or atypical chest pain.

In another group, the main complaint was dysphagia and gas bloating. Assessment of dysphagia was done using the dysphagia scoring scale and the average score was between 1-3 Table (1).

The preoperative workup in all patients included upper gastrointestinal endoscopy (UGE) and a barium study for visualization of the anatomical-morphological reason for failure. In addition, esophageal manometry and 24-hour ph monitoring were performed routinely in every patient preoperatively.

Diagnostic 24-h pH monitoring was performed according to a standardized procedure after manometric determination of the LES position. The $\mathrm{pH}$ electrode then was positioned $5 \mathrm{~cm}$ above the upper border of the LES for evaluation of esophageal acid exposure. Patients were instructed to stop potential intake of $\mathrm{H} 2$ blockers or proton pump inhibitors at least 1 week before monitoring. The existence of pathological acid reflux was defined as a DeMeester score13 >14.72.

The type of refundoplication was tailored to the results of esophageal manometry. $360^{\circ}$ Nissen fundoplication was performed in all patients with normal esophageal motility, whereas patients with poor esophageal motility (a pressure of $<30 \mathrm{~mm} \mathrm{Hg}$ in the lower esophageal segments in response to wet swallows) or disordered peristalsis ( $>40 \%$ simultaneous contractions in wet swallows) underwent $270^{\circ}$ Toupet fundoplication or floppy Nissen fundoplication.

Operative technique: Revision antireflux surgery was more difficult than primary surgery for reflux, particularly if the previous procedure was performed using an open technique. Open procedure was our protocol in recurrent surgery.

Anathesia: The operation was done under general endotracheal anesthesia with muscle relaxant.

Positioning and instrumentation: The patient was placed on the operating table in the supine position. The operating table was placed in a reverse Trendelenburg position. Excellent exposure of the esophageal hiatus was paramount in performing an open procedure. This was achieved by utilizing an upper hand retractor fit with two blades for the right and left costal margins. Extra-long surgical instruments were usually needed for the operative procedure, especially when operating on men and obese patients.

Surgical steps: An upper midline abdominal incision was used for access. Proximally, this extended between the xiphisternum and the left costal margin, and distally it finished just above or just below the umbilicus.

a) Hiatal Dissection: Upper abdominal adhesions were divided to expose the operative field. The left lobe of the liver was usually adherent to the anterior wall of the upper 
stomach and the previous fundoplication. This was first separated from the stomach to expose the hiatal region.

The left lobe of the liver was displaced forward and to the right to expose the upper stomach and the region of the esophageal diaphragmatic hiatus. It was not necessary to divide the triangular ligament of the left lobe of the liver to displace the lobe medially, except when the liver was enlarged.

The tissue planes in the hiatal region were often difficult to find, and the hiatal rim and esophagus were dissected with caution to avoid perforation of the esophagus or stomach. A nasogastric tube was passed to aid in the identification of the esophagus. It was important to mobilize the esophagus till the gastro-esophageal junction and the upper stomach. This was done anteriorly by dividing the adhesions from the stomach to the liver and diaphragm using sharp dissection. Posteriorly the lesser sac was entered and the stomach was dissected free from the pancreas and retroperitoneal tissues. The vagal nerve trunks were difficult to identify during revision and there was high incidence of division of at least one of them.

b) Identification of the previous fundoplication: It was only when the gastroesophagel junction and the proximal stomach were completely free that any assessment was made to know the cause of failure. In 6 patients, recurrence of symptoms was due to intrathoracic wrap migration and this required reduction of the herniated part. Restoration of the normal anatomy by fully reversing and breaking down the previous fundoplication.

c) Formation of a new fundoplication in cases of recurrent reflux: After breakdown of the wrap, the esophagus was mobilized and a retroesophageal window was created. The right and left crura and the crural commissure were dissected exactly. After exact identification of the hiatal crura, crural closure was performed using interrupted 2-0 non-absorbable polyprolene sutures. After closing the crura posteriorly, the esophagus was lying loose in the hiatus. An oval sheet was cut out of a $10 \times 15-\mathrm{cm}$ polypropylene mesh which we normally use for hernial repair. For the esophageal body, a 3- to 4-cm keyhole in the center of the oval mesh was cut out. After bringing the mesh intra-abdominally, it was placed around the esophagus at the gastroesophageal junction, so that the esophageal body was lying through the keyhole of the mesh. The circular mesh was fixed onto the diaphragm by interrupted 2-0 non-absorbable polypropylene sutures.

It was usually necessary to make sure that all short gastric vessels were ligated and divided in order to have enough stomach to carry out a good fundoplication Figure (1).

The stomach was then grasped with two pairs of long non traumatic Babcock forceps, and manipulated to ensure that it came around the back of the esophagus loosely. If the stomach was tight, and did not sit comfortably without undue tension, the Babcock forceps was progressively adjusted and a looser piece of stomach grasped. This was continued until a satisfactorily loose piece of stomach was identified. Three interrupted 2-0 nonabsorbable polyprolene sutures were placed to form the fundoplication (either Nissen or Toupet). The sutures were passed deeply through the serosa and muscle of the stomach wall on both sides, and also more superficially through the muscle of the esophageal wall.

d) Formation of a new fundoplication in cases of dysphagia: The hiatus was routinely repaired without mesh (except in one patient who was having para-oesophageal herniation) using posteriorly placed sutures. These should narrow the hiatus to approximately $2.5 \mathrm{~cm}$ in diameter. To do this, the reconstructed hiatal rim sat loosely (not tightly) around the esophagus with a 52 Fr bougie sited across the gastroesophageal junction. At the time of Nissen fundoplication a 56 Fr bougie was advanced orally through the hiatus by the anesthesiologist.

Follow-up : Arrangements were made for clinical follow-up review in the out patient clinic at 3, 6 and 12 months. Objective follow-up investigations through 24-hour $\mathrm{pH}$ monitoring and esophageal manometry were performed $6 \& 12$ months later.

Statistical methodology: Data analysis was 
performed with SPSS version 12.0 (SPSS, Chicago,IL). Frequencies were used to describe statistics for qualitative categorical variables. Nonparametric variables are expressed as median (range), and normally distributed variables are expressed as mean ( \pm standard deviation), Paired t-test was used to compare quantitative variable in the same group, $\mathrm{P}$ value $>0.05$ insignificant $\mathrm{P}<0.05$ significant $\mathrm{P}<0.01$ highly significant.

\section{Results:}

31 patients entered the study, 14 (45.1\%) of them were males and 17 (54.9) were females Table (2), ranging from 27 to 52 years (mean age $39.44 \pm 7.5)$. Eleven patients (35.5\%) with previous laparoscopic fundoplication and twenty patients (64.5\%) with previous open fundoplication.

These patients were classified into two groups. The 1st group who complained of recurrence of symptoms and the 2nd group who complained of symptoms of overcorrection like dysphagia and gas bloating syndrome Table (3).

Redo surgery was performed to 12 out of 18 patients from the recurrence group and 4 patients out of 13 from the dysphagia group. The operating time varied between 120 and 230 min depending on the extent of intraabdominal adhesions; there were no intraoperative complications.

As regard the recurrence group they were 18 from 31 (58.1\%), 8 males and 10 females. 2 patients from the recurrence group were also complaining of dysphagia. 12 pattients underwent intially Nissen fundoplication (66.7\%) and 6 patients underwnt initially Toupet fundoplication (33.3\%).

Medical treatment started first which consisted mainly of proton pump inhibitors and life style modification for 3-5 months and this was very effective only in 6 patients and all were having Nissen fundoplication.

Investigations were done for the other 12 patients of the recurrence group who failed to have complete relief of symptoms on medical treatment and included UGE, barium study, 24-hours $\mathrm{pH}$ monitoring and esophageal manometry.
Barium study revealed intrathoracic wrap migration in 6 patients Figure (2), UGE revealed oesophagitis in all of them; $\mathrm{pH}$ monitoring revealed DeMeester score between 52 and 56 (mean $54.0 \pm 8.3$ ). Esophageal manometry revealed lower oesophageal sphincter (LES) pressure

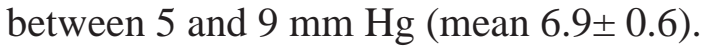

Intra-operative findings in the 12 patients revealed intrathoracic wrap migration in 6 patients (50\%), fundoplication disruption in 4 patients (33.3\%) due to poor ligation and division of the short gastric vessels, and slipped fundoplication in 2 patients (16.7\%) Table (4), Figures $(3,4)$.

Nissen or Toupet refundoplication was done according to the pre-operative manometric findings as mentioned before.

As regard the dysphagia group they were 13 from 31 (41.9\%), 5 males and 8 females. 12 pattients (92.3\%) underwent intially Nissen fundoplication and 1 patient (7.7\%) underwnt initially Toupet fundoplication.

The 13 patients were treated with dietary modification and reassurance, the dysphagia resolved spontaneously within 2-3 months in 5 patients out of 13 . However 8 patients experienced dysphagia that persisted beyond 3 months.

Investigations were done in these 8 patients and included UGE, barium study, 24-hours $\mathrm{pH}$ monitoring and esophageal manometry. UGE and barium revealed that the previous fundoplication had resulted in a mechanical obstruction of the lower esophagus in 5 patients; barium revealed para-oesophageal herniation in 1 patient. Esophageal manometry revealed resting LES

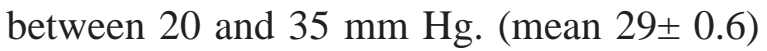
in the 8 patients. 24-hours $\mathrm{pH}$ monitoring was normal in all.

7 out of the above mentioned 8 patients (apart from the patient with para-oesophageal herniation) required a single session of dilatation with polyvinyl bougies to a mean diameter of $18 \mathrm{~mm}$ (54 Fr gauge). This was very effective in 3 out of 7 patients. 2 months later, the remaining 4 patients underwent another session of pneumatic dilatation using $30 \mathrm{~mm}$ to $40 \mathrm{~mm}$ diameter balloons; 


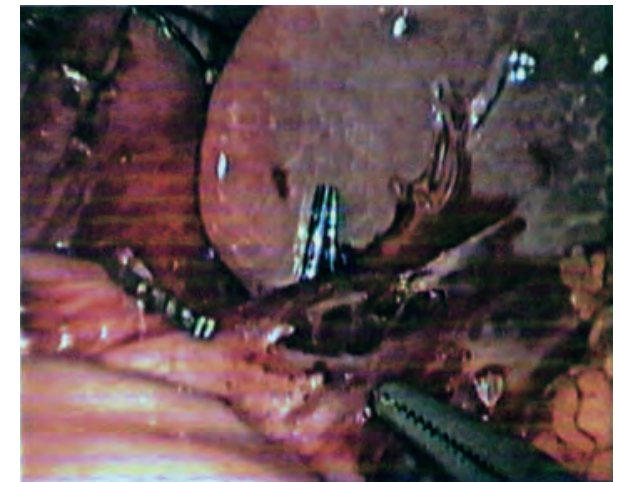

Figure (1): Ligation and division of short gastric vessels.

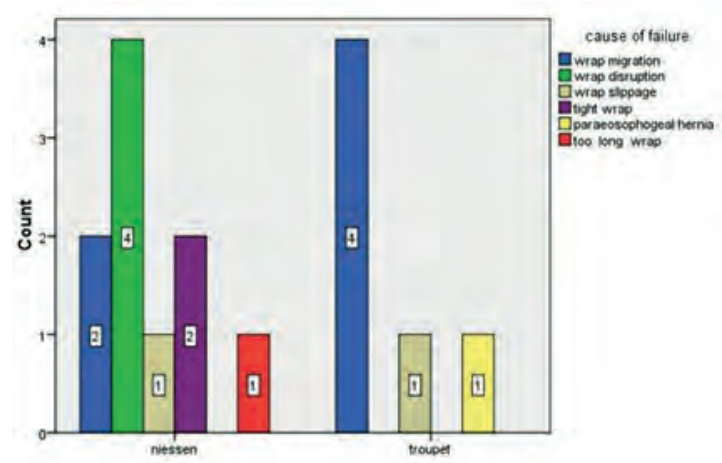

Figure (3): Causes of failure of anti-reflux surgery in our study.

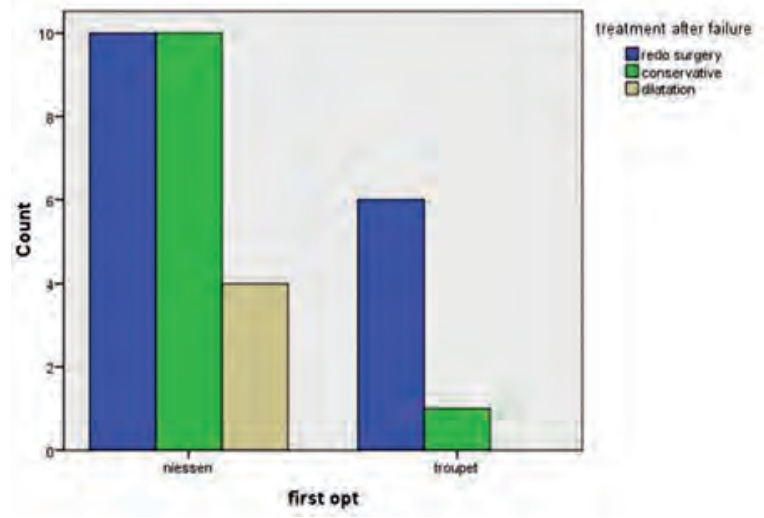

Figure (5): shows the type of treatment in relation to the $1^{\text {st }}$ fundoplication.

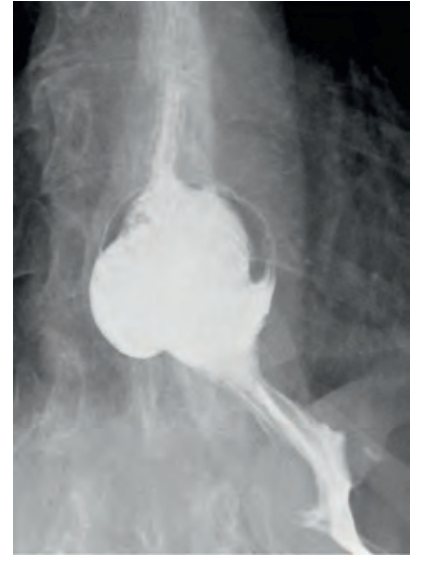

Figure (2): Barium study showing intrathoracic wrap migration.

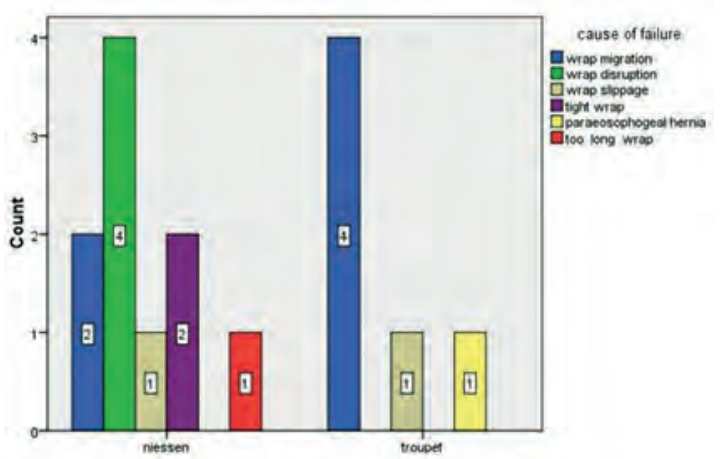

Figure (4): Cause of failure in relation to the first fundoplication.
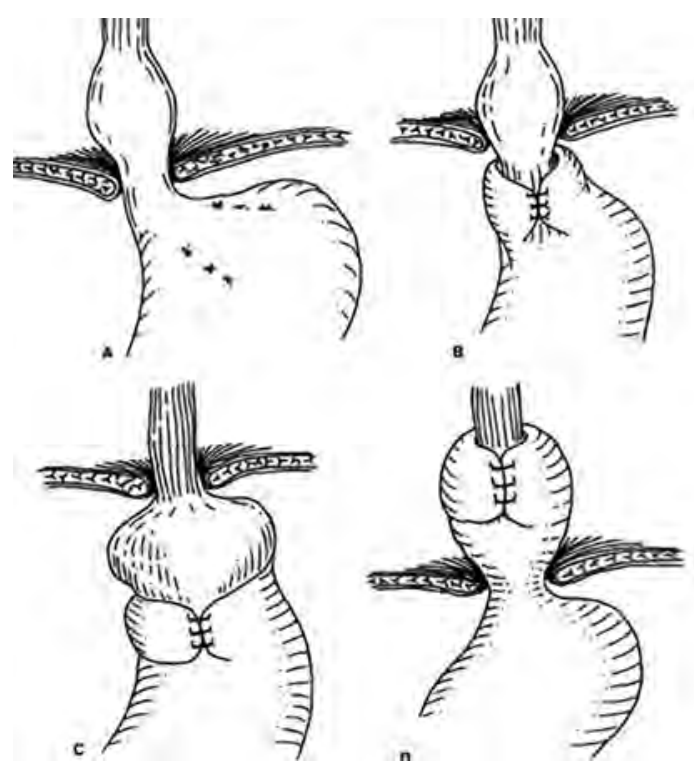

Figure (6): Types of fundoplication failures. A: Disrupted wrap. $B \quad \& \quad C$ : Slipped fundoplication. D: Intrathoracic migration of the fundoplication.

para-oesophageal herniation.

Intra-operative, a para-oesophageal hiatus hernia was a cause of post-operative dysphagia 
Table (1) : Scoring system for dysphasia.

\begin{tabular}{|l|l|}
\hline Score & \multicolumn{1}{|c|}{ Interpretation } \\
\hline 0 & Able to consume a normal diet \\
\hline 1 & Dysphagia with certain solid foods \\
\hline 2 & Able to swallow semi-solid soft foods \\
\hline 3 & Able to swallow liquids only \\
\hline 4 & Unable to swallow saliva (complete dysphagia) \\
\hline
\end{tabular}

Table 2 : Sex distribution.

\begin{tabular}{|l|l|l|}
\hline & Frequency & Percent \\
\hline Male & 13 & 41.9 \\
\hline Female & 18 & 58.1 \\
\hline Total & 31 & 100.0 \\
\hline
\end{tabular}

Table 3 : Type of failure.

\begin{tabular}{|l|l|l|}
\hline \multicolumn{1}{|c|}{ Valid } & Frequency & Percent \\
\hline Recurrence & 18 & 58.1 \\
\hline Dysphagia & 13 & 41.9 \\
\hline Total & 31 & 100.0 \\
\hline
\end{tabular}

Table 4 : Showing causes of failure of anti-reflux failure in our study.

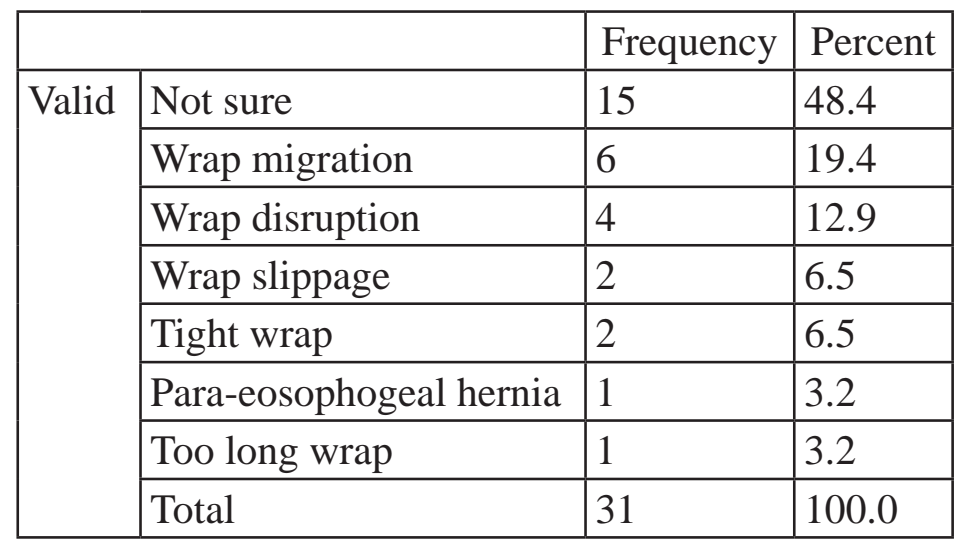

Table 5 : Showing summary of treatment after failure.

\begin{tabular}{|l|l|l|}
\hline & Frequency & Percent \\
\hline Redo surgery & 16 & 51.6 \\
\hline Conservative & 11 & 35.5 \\
\hline Dilatation & 4 & 12.9 \\
\hline Total & 31 & 100.0 \\
\hline
\end{tabular}

as mentioned before in one patient, too tight fundoplication was found in 2 patients and too long fundoplication $(4 \mathrm{~cm})$ in 1 patient.
Floppy Nissen was done for these 4 patients Tables (4) Figures $(3,4)$.

Table (5) shows that 16 patients out of 
Table 6 : Showing treatment after failure in relation to the type of first surgery.

\begin{tabular}{|l|l|l|l|l|l|l|l|l|}
\hline \multicolumn{2}{|c|}{} & Redo surgery & Conservative & Dilatation & Total & Chi square & P value & Sig \\
\hline \multirow{2}{*}{$\begin{array}{l}\text { First } \\
\text { operation }\end{array}$} & Nissen & 10 & 10 & 4 & 24 & 4.349 & 0.114 & NS \\
\cline { 2 - 6 } & Toupet & 6 & 1 & 0 & 7 & & & \\
\cline { 1 - 7 } Total & 16 & 11 & 4 & 31 & & & \\
\hline
\end{tabular}

Table (7) : Lists the causes of persistent postfundoplication dysphagia. ${ }^{13}$

\begin{tabular}{|l|l|}
\hline a) Mechanical obstruction resulting from the & $\begin{array}{l}\text { 1. Too long fundoplication } \\
\text { fundoplication procedure }\end{array}$ \\
& 2. Too tight fundoplication \\
& 3. Slipped fundoplication \\
4. Crural repair constricting the oesophagus & 5. Para-oesophageal herniation \\
\hline b) GERD-related & $\begin{array}{l}\text { 6. Peptic oesophageal stricture } \\
\text { 7. Recurrent reflux oesophagitis }\end{array}$ \\
\hline c) Esophageal motility disorder & 8. Ineffective oesophageal motility \\
& 9. Achalasia missed pre-operatively \\
\hline
\end{tabular}

$31(51.6 \%)$ with failed anti-reflux surgery were in need for revision of fundoplication through a redo surgery, 11 patients (35.5\%) were managed conservatively, and 4 patients (12.9\%) required endoscopic dilatation.

Table (6) shows the type of treatment after failed anti-reflux surgery in relation to the 1 st fundoplication and reveals that concerning the redo surgery there is no significance difference whether the patient had underwent Nissen or Toupet $(\mathrm{P}>0.05)$, however oesophageal dilatation was more common with Nissen fundoplication Figure (5).

As regard the recurrence group : Significant improvement in heart burn and regurgitation was noted in this group, with no difference between those who underwent Nissen refundoplication and those who underwent Toupet. However 3 patients experienced mild dysphagia to solids in those who underwent Nissen fundoplication that was managed conservatively and disappeared after 3 months.

One patient had a gastric perforation which was diagnosed on the 3rd postoperative day, the patient was re-explored with closure of the perforation over a gastrostomy tube and insertion of a feeding jejunostomy tube.

Objective follow up: 24-hour $\mathrm{pH}$ monitoring: Esophageal acid exposure improved significantly after surgery. The preoperative mean DeMeester score of $54.0 \pm$ 8.3 decreased significantly to $14.7 \pm 3.8$ at 6 months $(p=0.001)$ and to $13.8 \pm 5.4$ at 1 year.

Esophageal manometry: The pre-operative mean resting LES pressure of $6.9 \pm 0.6 \mathrm{~mm}$ $\mathrm{Hg}$ increased to $13.6 \pm 0.6$ after 6 months and to $13.1 \pm 0.4$ after 1 year.

As regard the dysphagia group : Significant improvements in dysphagia was noted in this group.

Esophageal manometry : The preoperative mean resting LES pressure of mean $29 \pm 0.6 \mathrm{~mm} \mathrm{Hg}$ has decreased to $16.6 \pm 0.4$ after 6 months and to $15.5 \pm 0.8$ after 1 year.

\section{Discussion:}

Fundoplication either Nissen or Toupet is the most popular of the anti-reflux procedures in patients with GERD. Failure of fundoplication occurs when the patient, after the repair, experiences persistent or recurrent reflux symptoms, or develops new symptoms as inability to swallow normally, or suffers from upper abdominal discomfort or other gastrointestinal symptoms. The assessment of these symptoms and the selection of patients who need further surgery remains a challenging problem. ${ }^{4}$

Persistent or recurrent postoperative reflux 
symptoms are usually due intrathoracic wrap migration, wrap disruption, slipped fundoplication, twisting of the fundoplication and improper construction of the wrap using the body rather than the fundus of the stomach $^{5}$ Figure (6).

Intrathoracic wrap migration can occur due to inadequate crural closure, disruption of the crural repair, insufficient esophageal mobilization, shortened esophagus or severe retching soon after surgery. Recent studies recommend prosthetic hiatal closure in cases of recurrence. ${ }^{6}$

Garnderath et $\mathrm{al}^{7}$ showed that in a study that included 33 patients who underwent laparoscopic refundoplication for recurrent symptoms of GERD after primary failed laparoscopic or open antireflux surgery, the underlying morphological complication for symptom recurrence in all patients was intrathoracic migration of the fundoplication.

In our study 6 patients out of 12 patients who underwent redo for recurrent symptoms were having a migrated fundoplication.

Wrap disruption is one of the most common causes of failure. It frequently occurs early during the postoperative course. This reflects the widespread use of absorbable suture material when creating the wrap, inadequate suture technique (i.e., taking inadequate bites of tissue) and insufficient mobilization of the fundus may also contribute to wrap disruption. ${ }^{8}$

In our study 4 patients out of 12 patients who underwent redo for recurrent symptoms were having a wrap disruption.

A slipped fundoplication "the so-called telescope phenomenon" can occur in two ways: (1) The fundoplication is fashioned in the correct location, but a portion of the stomach later herniates "slips" through the fundoplication; or (2) The surgeon mistakes the proximal stomach for the distal oesophagus, and inadvertently fashions the fundoplication around the stomach. Although the later situation represents a technical surgical error rather than a true slippage, the condition is called a slipped fundoplication despite the misnomer.

Type (1) slippage usually occurs if the sutures are not passed through the esophageal wall. Type (2) slippage occurs due to unrecognized esophageal shortening and inadequate mobilization of the esophagus leading to a wrapping of stomach around stomach, rather than stomach around the lower esophagus. Patients with slipped fundoplication usually complain of both heartburn and dysphagia occurring after a symptom free postoperative interval. ${ }^{9}$

In our study 2 patients were having a slipped fundoplication and they were complaining of both heart burn and dysphagia but the most annoying complaint was recurrence of heart burn and that's why they were categorized in the recurrence group.

For patients with persistent or recurrent symptoms after fundoplication, endoscopy can answer several important questions. (1) Is there reflux oesophagitis? The presence of reflux oesophagitis provides objective evidence that the operation has not controlled GERD. (2) Is there any another lesion that can explain the symptom as gastric or duodenal ulcer. (3) Does the fundoplication appear to be anatomically correct? Fundoplication creates characteristic folds in the proximal stomach that usually can be seen best with the endoscope in the retroflexed position. If the folds are seen above the diaphragm, it indicates wrap migration. If there is a pouch of the stomach proximal to the folds of fundoplication, it indicates slipped fundoplication. Finally, the absence of fundoplication folds suggests total disruption of the wrap. ${ }^{10}$

In our study, all patients who underwent redo for recurrent symptoms were having oesophagitis, and were negative for peptic ulcer but unfortunately the endoscopist did not aid us to identify the anatomical or morphological problem of the failed wrap.

Ambulatory monitoring of esophageal $\mathrm{pH}$ is also an important diagnostic tool for patients with persistent or recurrent symptoms after fundoplication, the demonstration of abnormal acid reflux and the correlation of symptoms with reflux episodes establish that the operation has failed at its primary goal. However para-oesophageal herniation and 
anatomical relationships among organs may be better appreciated by barium studies. 11

Approximately $50 \%$ of patients experience dysphagia immediately after fundoplication, presumably as a consequence of the edema and inflammation caused by surgery. The patients are treated by dietary modifications and reassurance, and the dysphagia usually resolves spontaneously within 2-3 months. ${ }^{12}$

In cases of postfundoplication mechanical dysphagia as a result of too long or too tight wrap or if the diaphragmatic crural repair constricts the oesophagus, dilatation can relive the dysphagia in $50-70 \%$ of cases. For patients who have dysphagia due to slipped fundoplication or para-oesophageal herniation, dilatation therapy usually will fail and re-operation will be necessary in most cases. 10

The creation of a too tight or too long wrap is manifested by persistent dysphagia starting shortly after the anti-reflux procedure. Postoperative manometry in these patients shows a high-pressure sphincter which does not relax on swallowing. ${ }^{12}$

In our study, dilation was effective in 4 out of 7 patients (57.2\%) who showed failure to conservative management of postoperative fundoplication dysphagia, this percentage is relatively low when compared with other studies.

In a similar study, dilation was successful to relieve dysphagia in 12 of 18 (67\%) patients. ${ }^{13}$

Summing up all available data and studies, failure rates of primary fundoplication range as high as $30 \% .{ }^{14}$ However, there is a lack of clarity concerning how failure is defined and what the therapeutic consequences should be. For example, what if the symptom is still present but only improved to the point that it is more tolerable or more readily controlled with medication? Is that a failure or an incomplete success ${ }^{15}$

In our own clinical practice, the usual definition of failure is a combination of recurrent or persistent gastroesophageal reflux disease (GERD) symptoms or appearance of new symptoms developing from an anatomical or morphological complication that usually needs a redo surgery.

\section{Conclusion :}

This analysis of the reasons for failure fundoplication indicates that several factors are essential for a successful outcome after fundoplication. These are: 1) a meticulous surgical technique: careful fundic mobilization, secure diaphragmatic closure, esophageal lengthening construction of a wrap of optimum length (not too long and not too short) better around a large bougie. 2) The identification and careful selection of patients who might benefit from anti-reflux surgery; and 3) a sound interpretation of the data taken from oesophageal manometry and 24-hours ambulatory $\mathrm{pH}$ monitoring. Attention to these factors will avoid failures in most instances.

\section{Reference:}

1- Wehrli H: Laparoscopic fundoplication for gastroesophageal reflux disease. Ther Umsch 2005; 62(2): 96-99.

2- Migliore M, Jeyasingham K: Re-operations for failed anti-reflux surgery. Lessons from the past and prospects for the future. Ann Ital Chir 2009; 80: 267-274.

3- Siewert JR, Isolauri J, Feussner H: Reoperation following failed fundoplication. World J Surg 1989; 13: 791.

4- Horgan S, Pohl D, Bogetti D, Eubanks T, Pellegrini C: Failed antireflux surgery. What have we learned from reoperations? Arch Surg 1999; 134: 809-817.

5- Warting JP: Management of postfundoplication complications. Semin Gastrointest Dis 1999; 10: 121-129.

6- Hunter JG, Smith CD, Branum GD, Waring JP, Trus TL, Cornwell M, et al: Laparoscopic fundoplication failures: Patterns of failure and response to fundoplication revision. Ann Surg. 1999; 230: 595-606.

7- Granderath F, Granderath U, Pointner R: Laparoscopic revisiona fundoplication with cicylar hiatal mesh prothesis: The long-term results. World J Surg 2008; 32: 999-1007.

8- O’Hanrahan T, Marples M. Bancewicz J: Recurrent reflux and wrap disruption after Nissen fundoplication: Detection, incidence, timing. Br J Surg 1990; 77: 545-547.

9- Siewert JR, Lepsien G, Weiser HF, Schattenmann G, Peiper HJ: Das TeleskopPhanomen Chirurg 1999; 48: 640. 
10- Johnson DA, Younes Z, Hogan WJ: Endoscopic assessment of hiatal hernia repair. Gastrointest Endosc 2000; 52: 650-659.

11- Stein HJ, DeMeester TR, Hinder RA: Outpatient physiologic testing and surgical management of foregut motility disorders. Cur Probl Surg 1992: 29: 415-455.

12- Donahue PE, Samelson S, Nyhus LN, Bombeck CT: The floppy Nissen fundoplication. Arch Surg 1985; 120: 663-668.

13- Malhi-Chowla N, Gorecki P, Bammer T, et al: Dilation after fundoplication: Timing, frequency, indications, and outcome.
Gastrointest Endosc 2002; 55 : 219-223.

14- Wo JM, Trus TL, Richardson WS, Branum GD, Mauren SJ, Waring JP: Evaluation and management of postfundoplication dysphagia. Am J Gastroenterol Nov 1996; 91(11): 2318-2322.

15- Johansson J, Peters JH: Preoperative evaluation of gastroesophageal reflux disease. Acta Chir Austriaca 1999; 31: 338-345.

16- Spechler SJ, Lee E, Ahnen D, et al: Long-term outcome of medical and surgical therapies for gastroesophageal reflux disease: Followup of randomized controlled trial. JAMA 2001; 285: 2331-2338. 